\title{
Universiteit
}

Leiden

The Netherlands

\section{Electromechanical noise in a diffusive conductor}

Shytov, A.V.; Levitov, L.S.; Beenakker, C.W.J.

\section{Citation}

Shytov, A. V., Levitov, L. S., \& Beenakker, C. W. J. (2002). Electromechanical noise in a diffusive conductor. Physical Review Letters, 88(22), 228303.

doi:10.1103/PhysRevLett.88.228303

Version: $\quad$ Not Applicable (or Unknown)

License: $\quad$ Leiden University Non-exclusive license

Downloaded from: https://hdl.handle.net/1887/64316

Note: To cite this publication please use the final published version (if applicable). 


\title{
Electromechanical Noise in a Diffusive Conductor
}

\author{
A. V. Shytov, ${ }^{1}$ L. S. Levitov, ${ }^{2}$ and C. W. J. Beenakker ${ }^{3}$ \\ ${ }^{1}$ Institute for Theoretical Physics, University of California, Santa Barbara, California 93106-4030 \\ ${ }^{2}$ Department of Physics, Center for Materials Science \& Engineering, Massachusetts Institute of Technology, \\ Cambridge, Massachusetts 02139 \\ ${ }^{3}$ Instituut-Lorentz, Universiteit Leiden, P.O. Box 9506, 2300 RA Leiden, The Netherlands
}

(Received 29 October 2001; published 17 May 2002)

\begin{abstract}
Electrons moving in a conductor can transfer momentum to the lattice via collisions with impurities and boundaries, giving rise to a fluctuating mechanical stress tensor. The root-mean-squared momentum transfer per scattering event in a disordered metal (of dimension $L$ greater than the mean-free path $l$ and screening length $\xi$ ) is found to be reduced below the Fermi momentum by a factor of order $l / L$ for shear fluctuations and $(\xi / L)^{2}$ for pressure fluctuations. The excitation of an elastic bending mode by the shear fluctuations is estimated to fall within current experimental sensitivity for a nanomechanical oscillator.
\end{abstract}

DOI: 10.1103/PhysRevLett.88.228303

Impressive advances in the fabrication of miniature mechanical oscillators provide new opportunities for research in mesoscopic physics [1,2]. The coupling of electrical and mechanical degrees of freedom is of particular interest. We mention the observation of thermal vibration [3] and acoustoelectric effects [4] in carbon nanotubes, the coupling of the center-of-mass motion of $\mathrm{C}_{60}$ molecules and single-electron hopping [5], and also theoretical work [6] on the coupling between a tunneling electrical current and a localized phonon mode.

This Letter was motivated by a question posed to us by M. Roukes: Electrons in a metal collide with impurities and thereby exert a fluctuating force on the lattice. In equilibrium this electromechanical force cannot be distinguished from other sources of thermal noise. Might it be measurable out of equilibrium by driving a current through a nanoscale oscillator? To address this question one has to consider a delicate balance of forces.

We will provide both a general theory and a specific application to the electromechanical excitation of a bending mode in the geometry of Fig. 1: a thin elastic beam connecting two massive Ohmic contacts. The beam could be a conductor or an insulator covered with a metal (e.g., a metallized suspended silicon beam [7]). We calculate the excess noise in the bending mode that arises in the presence of a de voltage $V$ and conclude that it should be observable in the background of the thermal noise.

Let us first discuss the order of magnitude. The noise at low temperatures is due to the $\mathcal{N} e V / E_{F}$ "noisy" electrons within a range $e V$ of the Fermi energy $E_{F}$ (with $\mathcal{N}$ the total electron number in the metal). Each electron transfers to the lattice a typical momentum $\Delta p \simeq p_{F}$ in a scattering time $\tau$. The mean-squared momentum transfer in a time $t$ for uncorrelated increments $\Delta p$ would be

$$
\left(\mathcal{N} e V / E_{F}\right)(\Delta p)^{2}(t / \tau) \simeq \mathcal{N} m^{*} e V(t / \tau) \equiv \mathcal{P}_{\max } t
$$

with $m^{*}$ the electron effective mass.
PACS numbers: $85.85 .+\mathrm{j}, 73.23 .-\mathrm{b}, 73.50 . \mathrm{Td}, 77.65 .-\mathrm{j}$

We find that $\mathcal{P}_{\max }$ overestimates the fluctuations in the transverse force. The actual noise power is of order $\mathcal{P} \simeq$ $(l / L)^{2} \mathcal{P}_{\max }$, with $L$ the length of the beam and $l$ the meanfree path in the metal. The reduction appears because subsequent momentum transfers are strongly correlated, since an electron being scattered back and forth alternatingly transfers positive and negative momentum to the lattice. The factor $(l / L)^{2}$ reduces the noise substantially, but we estimate that it should be observable in an oscillator with a $10^{-16} \mathrm{~N} / \sqrt{\mathrm{Hz}}$ sensitivity [2,7].

We combine two independently developed theoretical frameworks: the dynamic theory of elasticity $[8,9]$ and the kinetic theory of fluctuations [10]. We start from the Boltzmann-Langevin equation of Kogan and Shulman [10]. This is a kinetic equation with a fluctuating source $\delta J(\mathbf{r}, \mathbf{p}, t)$ that describes fluctuations in the time of the distribution function $n(\mathbf{r}, \mathbf{p}, t)$,

$$
\left(\partial_{t}+\mathbf{v} \cdot \nabla_{\mathbf{r}}+e \mathbf{E} \cdot \nabla_{\mathbf{p}}+S\right) n=\delta J .
$$

Here $\mathbf{p}=m^{*} \mathbf{v}$ is the quasimomentum and $\mathbf{E}(\mathbf{r}, t)$ is the electric field. The collision integral $S$ for elastic scattering on impurities (with rate $W$ ) is given by

$$
S n(\mathbf{p})=\left\langle W\left(\hat{\mathbf{p}} \cdot \hat{\mathbf{p}}^{\prime}\right)\left[n(\mathbf{p})-n\left(\mathbf{p}^{\prime}\right)\right]\right\rangle_{\hat{\mathbf{p}}^{\prime}} \cdot
$$

The angular brackets indicate an average over the direction $\hat{\mathbf{p}}^{\prime}$ of the momentum $\mathbf{p}^{\prime}$, with $\left|\mathbf{p}^{\prime}\right|=|\mathbf{p}|$.

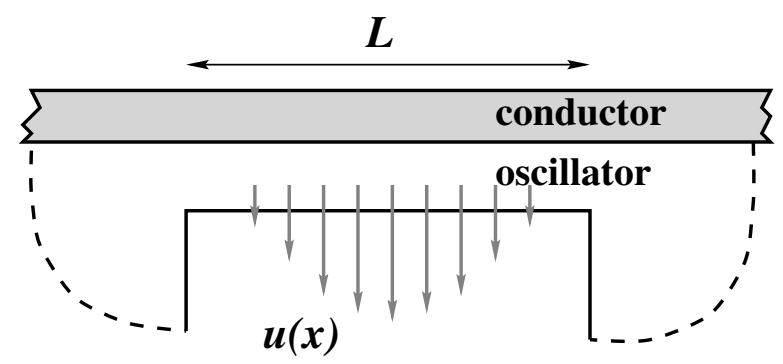

FIG. 1. Sketch of an elastic beam clamped at both ends to a contact and covered by a metal layer. A current flowing through the metal excites a bending mode $u(x)$ of the beam. 
The noise source $\delta J$ has zero time average and variance

$$
\begin{aligned}
\overline{\delta J(\mathbf{r}, \mathbf{p}, t) \delta J\left(\mathbf{r}^{\prime}, \mathbf{p}^{\prime}, t^{\prime}\right)}= & \delta\left(\mathbf{r}-\mathbf{r}^{\prime}\right) \delta\left(t-t^{\prime}\right) \delta\left(\varepsilon-\varepsilon^{\prime}\right) \nu^{-1} \\
& \times\left[4 \pi \delta\left(\hat{\mathbf{p}}-\hat{\mathbf{p}}^{\prime}\right)\left\langle W\left(\hat{\mathbf{p}} \cdot \hat{\mathbf{p}}^{\prime \prime}\right)\left(\bar{n}+\bar{n}^{\prime \prime}-2 \bar{n} \bar{n}^{\prime \prime}\right)\right\rangle_{\hat{\mathbf{p}}^{\prime \prime}}-W\left(\hat{\mathbf{p}} \cdot \hat{\mathbf{p}}^{\prime}\right)\left(\bar{n}+\bar{n}^{\prime}-2 \bar{n} \bar{n}^{\prime}\right)\right] .
\end{aligned}
$$

Here $\nu(\varepsilon)$ is the density of states (at energy $\varepsilon=p^{2} / 2 m^{*}$ ) and $\bar{n}$ is the time-averaged distribution. We have set Planck's constant $h \equiv 1$, so that $n$ is dimensionless, and have abbreviated $\bar{n}^{\prime}=\bar{n}\left(\mathbf{r}, \mathbf{p}^{\prime}, t\right), \bar{n}^{\prime \prime}=\bar{n}\left(\mathbf{r}, \mathbf{p}^{\prime \prime}, t\right)$.

The force density $\mathbf{f}(\mathbf{r}, t)$ exerted by the electrons on the lattice is the divergence of a symmetric tensor $\boldsymbol{\Pi}$ that can be decomposed into an isotropic pressure $\Pi_{0}$ and a traceless shear tensor $\mathbf{\Sigma}$ :

$$
f_{\alpha}=-\nabla_{\beta} \Pi_{\alpha \beta}, \quad \Pi_{\alpha \beta}=\Pi_{0} \delta_{\alpha \beta}+\Sigma_{\alpha \beta} .
$$

In the approximation of a deformation-independent effective mass, one has $[9,11]$

$$
\Pi_{\alpha \beta}=m^{*} \int d \mathbf{p} v_{\alpha} v_{\beta} n
$$

The time-averaged force (5) vanishes, since it contains a derivative of the spatially uniform time-averaged distribution $\bar{n}$. The electrical current drag on impurities (the so-called "wind force") is cancelled by the electric field force exerted on the ions [12]. Since $\mathbf{f}$ is a total derivative the net fluctuating force vanishes as well at low frequencies (ignoring boundary contributions). Although the center of mass does not move, there are fluctuating compression modes (driven by $\Pi_{0}$ ) as well as torsion and bending modes (driven by $\mathbf{\Sigma}$ ). The driving force $\mathcal{F}(t)$ for each of these modes is obtained by weighing $\mathbf{f}(\mathbf{r}, t)$ with a sensitivity function $\mathbf{g}(\mathbf{r})$ proportional to the displacement field of the mode,

$$
\mathcal{F}=\int d \mathbf{r} \mathbf{f} \cdot \mathbf{g}=\int d \mathbf{r}\left(\Pi_{0} \nabla \cdot \mathbf{g}+\Sigma_{\alpha \beta} \frac{\partial g_{\beta}}{\partial r_{\alpha}}\right) .
$$

The two contributions $\Pi_{0}$ and $\boldsymbol{\Sigma}$ can be separated by expanding $n(\mathbf{r}, \mathbf{p}, t)$ in spherical harmonics $\mathbf{n}^{(q)}(\mathbf{r}, \varepsilon, t)$ with respect to the direction $\hat{\mathbf{p}}$ of the momentum. It is convenient to write the spherical harmonics in Cartesian (rather than spherical) coordinates,

$$
\begin{aligned}
n & =\sum_{q=0}^{\infty} \overline{\hat{p}_{\alpha_{1}} \cdots \hat{p}_{\alpha_{q}}} n_{\alpha_{1} \cdots \alpha_{q}}^{(q)} \\
& =n^{(0)}+\hat{p}_{\alpha} n_{\alpha}^{(1)}+\left(\hat{p}_{\alpha} \hat{p}_{\beta}-\frac{1}{3} \delta_{\alpha \beta}\right) n_{\alpha \beta}^{(2)}+\ldots
\end{aligned}
$$

Here $\widehat{\hat{\mathbf{p}}}^{q}$ is the traceless part of the symmetric tensor $\hat{p}_{\alpha_{1}} \cdots \hat{p}_{\alpha_{q}}$. These tensors form an orthonormal set [13],

$$
\left\langle\overline{\hat{\mathbf{p}}^{n}} \overline{\hat{\mathbf{p}}}^{m}\right\rangle_{\hat{\mathbf{p}}}=\delta_{n m} \frac{m !}{(2 m+1) ! !} \Delta^{(m)} .
$$

The tensor $\boldsymbol{\Delta}^{(m)}$ projects onto the traceless symmetric part of a tensor of rank $m$. We will need $\Delta_{\alpha \beta}^{(1)}=\delta_{\alpha \beta}$ and

$\Delta_{\alpha \beta \alpha^{\prime} \beta^{\prime}}^{(2)}=\frac{1}{2} \delta_{\alpha \alpha^{\prime}} \delta_{\beta \beta^{\prime}}+\frac{1}{2} \delta_{\alpha \beta^{\prime}} \delta_{\beta \alpha^{\prime}}-\frac{1}{3} \delta_{\alpha \beta} \delta_{\alpha^{\prime} \beta^{\prime}}$.

In view of the orthogonality of different spherical harmonics, one has

$$
\Pi_{0}=\frac{1}{3} \int d \varepsilon 2 \varepsilon \nu n^{(0)}, \quad \Sigma_{\alpha \beta}=\frac{2}{15} \int d \varepsilon 2 \varepsilon \nu n_{\alpha \beta}^{(2)} .
$$

The two harmonics $n^{(0)}$ and $\mathbf{n}^{(2)}$ have to be found from the kinetic equation (2). We first consider the harmonic $\mathbf{n}^{(2)}$ that determines the shear tensor $\mathbf{\Sigma}$, and then discuss the harmonic $n^{(0)}$ and resulting pressure $\Pi_{0}$.

To obtain an equation for $\mathbf{n}^{(2)}$ we multiply both sides of Eq. (2) by $\widehat{\hat{\mathbf{p}} \hat{\mathbf{p}}}$ and perform an angular average. Employing the diffusion approximation on length and time scales larger than $l$ and $\tau$, we neglect the derivatives with respect to $t$ and $\mathbf{r}$ in Eq. (2). Also, in the linear response approximation, we neglect the derivative with respect to $\mathbf{p}$, since it gives a term bilinear in $\mathbf{E}$ and $\mathbf{j}$. What remains is a local relation between $\mathbf{n}^{(2)}$ and the second harmonic $\mathbf{J}^{(2)}$ of the fluctuating source,

$$
\mathbf{n}^{(2)}=\tau_{2} \delta \mathbf{J}^{(2)} .
$$

The momentum transport time $\tau_{2}$ is defined by

$$
\frac{1}{\tau_{2}}=\frac{3}{4} \int_{-1}^{1} d \xi W(\xi)\left(1-\xi^{2}\right) .
$$

For anisotropic scattering the time $\tau_{2}$ is larger than the charge transport time $\tau$, defined by $\frac{1}{\tau}=$ $\frac{1}{2} \int_{-1}^{1} d \xi W(\xi)(1-\xi)$. (For isotropic scattering $\tau_{2}=$ $\tau=1 / W$.) The correlator of $\delta \mathbf{J}^{(2)}$ follows in the same way from Eq. (4). In the diffusion approximation we replace $\bar{n}, \bar{n}^{\prime}$, and $\bar{n}^{\prime \prime}$ by $\bar{n}^{(0)}$. Using Eq. (9) we arrive at

$$
\overline{\delta \mathbf{J}_{\alpha \beta}^{(2)}(\mathbf{r}, \varepsilon, t) \delta \mathbf{J}_{\alpha^{\prime} \beta^{\prime}}^{(2)}\left(\mathbf{r}^{\prime}, \varepsilon^{\prime}, t^{\prime}\right)}=\frac{15}{\nu \tau_{2}} \boldsymbol{\Delta}_{\alpha \beta \alpha^{\prime} \beta^{\prime}}^{(2)} \delta\left(t-t^{\prime}\right) \delta\left(\mathbf{r}-\mathbf{r}^{\prime}\right) \delta\left(\varepsilon-\varepsilon^{\prime}\right) \bar{n}^{(0)}(\mathbf{r}, \varepsilon)\left[1-\bar{n}^{(0)}(\mathbf{r}, \varepsilon)\right] .
$$

Since $\bar{n}^{(0)}(\mathbf{r}, \varepsilon)$ differs from 0 or 1 only in a narrow range near the Fermi level, we ignore the energy dependence of $\nu$ and $\tau_{2}$ and evaluate them at $\varepsilon=E_{F}$.

We quantify the transverse momentum noise through the correlator of the shear tensor,

$$
C_{\alpha \beta \alpha^{\prime} \beta^{\prime}}^{(2)}\left(\mathbf{r}, \mathbf{r}^{\prime}\right)=2 \int_{-\infty}^{\infty} d t \overline{\Sigma_{\alpha \beta}(\mathbf{r}, 0) \Sigma_{\alpha^{\prime} \beta^{\prime}}\left(\mathbf{r}^{\prime}, t\right)}
$$


Combining Eqs. (11), (12), and (14), we obtain the result

$$
\begin{aligned}
\boldsymbol{C}^{(2)}\left(\mathbf{r}, \mathbf{r}^{\prime}\right) & =\frac{8}{15}\left(m^{*} v_{F}^{2}\right)^{2} \tau_{2} \nu \delta\left(\mathbf{r}-\mathbf{r}^{\prime}\right) K(\mathbf{r}) \Delta^{(2)}, \\
K(\mathbf{r}) & =\int d \varepsilon \bar{n}^{(0)}(\mathbf{r}, \varepsilon)\left[1-\bar{n}^{(0)}(\mathbf{r}, \varepsilon)\right] .
\end{aligned}
$$

The kernel $K$ is given by [14] $K=(e V / L) x(1-x / L)$, where $V$ is the voltage applied between the two contacts at $x=0, L$. The parabolic profile $K(x)$ requires $k_{B} T \ll e V$ and the absence of inelastic scattering.

We now turn to the pressure fluctuations. Instead of Eqs. (12) and (14) we have the fluctuating drift-diffusion equation [14]

$$
\begin{gathered}
\mathbf{j}+D \nabla \rho-\sigma \mathbf{E}=e \tau \int d \varepsilon \nu v \delta \mathbf{J}^{(1)} \equiv \delta \mathbf{I}, \\
\overline{\delta I_{\alpha}(\mathbf{r}, t) \delta I_{\beta}\left(\mathbf{r}^{\prime}, t^{\prime}\right)}=2 \sigma \delta_{\alpha \beta} \delta\left(\mathbf{r}-\mathbf{r}^{\prime}\right) \delta\left(t-t^{\prime}\right) K(\mathbf{r}),
\end{gathered}
$$

which relates the fluctuations in the charge density $\rho=$ $e \int d \varepsilon \nu n^{(0)}$ and the current density $\mathbf{j}=\frac{1}{3} e \int d \varepsilon \nu v \mathbf{n}^{(1)}$. Once we know the charge density fluctuations we can find the fluctuating pressure from

$$
\Pi_{0}=(D / \mu) \delta \rho,
$$

cf. Eq. (11). The diffusion constant is $D=\frac{1}{3} v_{F}^{2} \tau$, the conductivity $\sigma=e^{2} \nu D$, and the mobility $\mu=e \tau / \mathrm{m}^{*}$.

The correlator $C^{(0)}$ of the pressure fluctuations is defined as in Eq. (15), with $\Sigma$ replaced by $\Pi_{0}$. To close the problem we need the continuity equation, $\partial \rho / \partial t+\nabla \cdot \mathbf{j}=0$, and the Poisson equation, $\kappa \nabla \cdot \mathbf{E}=\delta \rho$ (with dielectric constant $\kappa$ ). The time derivative of $\rho$ in the continuity equation may be omitted in the low-frequency regime. The fluctuations in the electron density then obey

$$
D \nabla^{2} \delta \rho-(\sigma / \kappa) \delta \rho=\nabla \cdot \delta \mathbf{I} .
$$

The current fluctuations create a fluctuating charge dipole that is screened over a length $\xi=(\kappa D / \sigma)^{1 / 2}=$ $\left(\kappa / e^{2} \nu\right)^{1 / 2}$. On length scales $\gg \xi$, one may neglect the diffusion term in Eq. (21) and use the local relation [15] $\delta \rho=-(\kappa / \sigma) \nabla \cdot \delta \mathbf{I}$. Equations (19) and (20) then yield

$$
C^{(0)}\left(\mathbf{r}, \mathbf{r}^{\prime}\right)=\frac{4 \sigma \xi^{4}}{\mu^{2}} \frac{\partial}{\partial \mathbf{r}} \cdot \frac{\partial}{\partial \mathbf{r}^{\prime}} \delta\left(\mathbf{r}-\mathbf{r}^{\prime}\right) K(\mathbf{r}) .
$$

The next step is to use the results (16) and (22) to estimate the low-frequency noise power $\mathcal{P}=2 \int_{-\infty}^{\infty} d t \times$ $\overline{\mathcal{F}(0) \mathcal{F}(t)}$ of the fluctuating force $\mathcal{F}(t)$ that drives a particular oscillator mode [16]. To that end the correlator (15) is integrated over $\mathbf{r}$ and $\mathbf{r}^{\prime}$, weighted by the sensitivity function of the mode as in Eq. (7). For a bending mode we use Eq. (16), which gives the noise power

$$
\mathcal{P}^{(2)}=\frac{8}{15}\left(m^{*} v_{F}^{2}\right)^{2} \tau_{2} \nu \int d \mathbf{r} K(\mathbf{r}) \Delta_{\alpha \beta \alpha^{\prime} \beta^{\prime}}^{(2)} \frac{\partial g_{\beta}}{\partial r_{\alpha}} \frac{\partial g_{\beta^{\prime}}}{\partial r_{\alpha^{\prime}}} .
$$

For a compression mode in a metal of size $\gg \xi$ we use
Eq. (22) and find

$$
\mathcal{P}^{(0)}=\frac{4 \sigma \xi^{4}}{\mu^{2}} \int d \mathbf{r} K(\mathbf{r})|\nabla \nabla \cdot \mathbf{g}|^{2} .
$$

For an order of magnitude estimate, we take $K \simeq e V$, $\mathbf{g} \simeq 1$, and estimate spatial derivatives by factors $1 / L$, and the volume integral by a factor $\mathcal{V}$. For simplicity we assume isotropic impurity scattering, so that $\tau_{2}=\tau$. Then the noise power due to fluctuations in the shear tensor is of order $\mathcal{P}^{(2)} \simeq\left(m^{*} v_{F}^{2}\right)^{2} \tau \nu e V \mathcal{V} L^{-2}$, and the noise power due to pressure fluctuations is of order $\mathcal{P}^{(0)} \simeq$ $\sigma \xi^{4} \mu^{-2} e V \mathcal{V} L^{-4}$. It is instructive to write these two estimates in the same form, using $\sigma / e \mu=\frac{1}{3} m^{*} v_{F}^{2} \nu=$ $\mathcal{N} / \mathcal{V} \equiv n_{e}$, with $n_{e}$ the electron density. One finds

$$
\mathcal{P}^{(0)} \simeq(\xi / L)^{4} \mathcal{P}_{\max }, \quad \mathcal{P}^{(2)} \simeq(l / L)^{2} \mathcal{P}_{\max },
$$

with $\mathcal{P}_{\max }=\mathcal{N} m^{*} \mathrm{eV} / \tau$ being the noise power for independent momentum transfers mentioned earlier.

The experimental observation of the shear tensor fluctuations looks more promising than the observation of the pressure fluctuations, first, because $\xi$ is typically $\ll(l L)^{1 / 2}$ so that $\mathcal{P}^{(0)} \ll \mathcal{P}^{(2)}$, and, second, because a typical oscillator operates in a bending or torsion mode rather than in a compression mode. For that reason we will now limit the more quantitative calculation to $\mathcal{P}^{(2)}$. We consider a bending mode $u(x) \cos \omega_{0} t$ in the geometry of Fig. 1. The sensitivity function $g(x)=u(x) / u\left(x_{0}\right)$ equals the displacement (in the $y$ direction) normalized by the value at a reference point $x_{0}$. We choose $x_{0}=L / 2$, so that $\mathcal{F}$ is equivalent to a point force at the beam's center. Equation (23) now takes the form

$$
\mathcal{P}=\frac{4}{5} n_{e} p_{F}(l \mathcal{A} / L) \int_{0}^{L} \frac{d x}{L} K(x)\left[L g^{\prime}(x)\right]^{2},
$$

with $\mathcal{A}=\mathcal{V} / L$ the cross-sectional area of the metal layer.

The wave equation for transverse waves is biharmonic, $d^{4} u / d x^{4}=k^{4} u$. The solution for doubly clamped boundary conditions is [17]

$$
\begin{aligned}
u(x)= & (\sin k L-\sinh k L)(\cos k x-\cosh k x) \\
& -(\cos k L-\cosh k L)(\sin k x-\sinh k x),
\end{aligned}
$$

with the resonance condition $\cos k L \cosh k L=1$. We use the lowest resonance at $k L=4.73$. Substituting $K=$ $(e V / L) x(1-x / L)$ and integrating, we obtain the excess noise $\mathcal{P}=\frac{4}{5} n_{e} p_{F}(l \mathcal{A} / L) \times 0.83 \mathrm{eV}$. If we insert values typical for a metal, $n_{e}=10^{29} \mathrm{~m}^{-3}, p_{F}=10^{-24} \mathrm{Ns}, l=$ $100 \mathrm{~nm}$, and choose typical dimensions $\mathcal{A} / L=10 \mathrm{~nm}$, then the force spectral density at $V=1 \mathrm{mV}$ is $\mathcal{P}=$ $10^{-32} \mathrm{~N}^{2} / \mathrm{Hz}$, well above the thermal noise power at low temperatures (of order $10^{-34} \mathrm{~N}^{2} / \mathrm{Hz}$ at $T=1 \mathrm{~K}$ [2]).

It is instructive to apply the result (26) to a system in thermal equilibrium, when $K(x)=k_{B} T$ for all $x$. In this case an independent estimate of the noise $\mathcal{P}_{0}$ is provided by the fluctuation-dissipation theorem: $\mathcal{P}_{0}=4 k_{B} T M \omega_{0} / Q_{0}$, with $M$ the active mass of the oscillator and $1 / Q_{0}$ the electromechanical contribution to the inverse quality factor. 
Equation (26) gives

$$
\frac{1}{Q_{0}}=\frac{1}{5}(l / L)^{2} \frac{\mathcal{N} m^{*}}{M \omega_{0} \tau} \int_{0}^{L} \frac{d x}{L}\left[L g^{\prime}(x)\right]^{2} .
$$

This electromechanical quality factor might be measurable in a superconducting metal, as an increase in the overall quality factor when $T$ drops below the critical temperature. One can also calculate $Q_{0}$ directly as an "absorption of ultrasound" by conduction electrons [9], providing a consistency check on our analysis. However, the nonequilibrium noise (26) with an $x$-dependent kernel $K(x)$ cannot be obtained from acoustic dissipation. The electromechanical part (28) of the overall quality factor can be significant. We estimate $Q_{0} \simeq 5 \times 10^{4}$ for the above metal parameters with $L=1 \mu \mathrm{m}, \omega_{0} / 2 \pi=100 \mathrm{MHz}$, and $M / \mathcal{N}=1000 m_{\text {proton. }}$. This is comparable to the reported values $Q \simeq 10^{3}-10^{5}$ [2].

Before concluding we mention an altogether different mechanism for electromechanical noise, which is the coupling of a fluctuating surface charge $\delta q(t)$ on the metal to the electromagnetic environment. In the presence of an electric field $E_{0}$ between the metal surface and the substrate (e.g., due to a mismatch in work functions), the charge fluctuations will give rise to a fluctuating transverse force with noise power

$$
\mathcal{P}_{\mathrm{env}} \simeq E_{0}^{2} \overline{\delta q^{2}} \simeq E_{0}^{2} C^{2} \overline{\delta V^{2}} \simeq E_{0}^{2} C^{2} R \max \left(k_{B} T, e V\right) .
$$

Here $C$ is the capacitance to the ground and $R=L / \mathcal{A} \sigma$ is the resistance of the metal [18]. The ratio $\mathcal{P}_{\text {env }} / \mathcal{P}^{(2)} \simeq$ $\left(E_{0} C L^{2} / e l \mathcal{N}\right)^{2}$ is quite small for typical parameter values. The reason is that the environmental charge fluctuations are a surface effect, while the whole bulk of the metal contributes to $\mathcal{P}^{(2)}$. Although the noise per electron is small in $l / L$, the total noise power $\mathcal{P}^{(2)}$ is big due to the large number $\mathcal{N} e V / E_{F}$ of contributing electrons.

In summary, we have addressed the fundamental question of the excitation of an elastic mode in a disordered metal out of equilibrium, as a result of the fluctuating momentum that an electrical current transfers to the lattice. The effect is small but measurable. The characteristic linear dependence of the electromechanical noise on the applied voltage should distinguish it from other sources of noise. We believe that a measurement is not only feasible but worth performing. Indeed, the nonequilibrium electric current noise has proven to be a remarkably powerful tool in the study of transport properties [19], precisely because it contains information that is not constrained by the fluctuation-dissipation theorem. The noise considered here could play a similar role for mechanical properties.

We mention one such application. Just as electrical shot noise measures the effective charge of the carriers, mechanical noise could be used to measure their momentum. This should be most intriguing in 1D electron systems, such as quantum wires and nanotubes, where strong electron interaction invalidates the Fermi liquid description. Electromechanical noise could thus be employed to measure the Luttinger liquid equivalent of the Fermi momen- tum. We are not aware of any other technique that would allow us to do such a measurement.

This work was motivated by discussions with M. Roukes during the Nanoscience program at the Institute for Theoretical Physics in Santa Barbara. We thank M. Kindermann, Yu. V. Nazarov, and B. Spivak for discussions, and acknowledge support by the National Science Foundation under Grants No. PHY99-07949 and No. DMR98-08941 (MRSEC program), and by the Netherlands Science Foundation NWO/FOM.

[1] J. A. Sidles, J. L. Garbini, K. J. Bruland, D. Rugar, O. Züger, S. Hoen, and C.S. Yannoni, Rev. Mod. Phys. 67, 249 (1995).

[2] M.L. Roukes, Phys. World 14, 25 (2001); cond-mat/ 0008187.

[3] M. M. J. Treacy, T. W. Ebbesen, and J. M. Gibson, Nature (London) 381, 678 (1996).

[4] B. Reulet, A. Yu. Kasumov, M. Kociak, R. Deblock, I. I. Khodos, Yu. B. Gorbatov, V. T. Volkov, C. Journet, and H. Bouchiat, Phys. Rev. Lett. 85, 2829 (2000).

[5] H. Park, J. Park, A. K. L. Lim, E. H. Anderson, A. P. Alivisatos, and P. L. McEuen, Nature (London) 407, 57 (2000).

[6] M. F. Bocko, K. A. Stephenson, and R. H. Koch, Phys. Rev. Lett. 61, 726 (1988); B. Yurke and G. P. Kochanski, Phys. Rev. B 41, 8184 (1990); C. Presilla, R. Onofrio, and M. F. Bocko, Phys. Rev. B 45, 3735 (1992); N. F. Schwabe, A. N. Cleland, M. C. Cross, and M. L. Roukes, Phys. Rev. B 52, 12911 (1995).

[7] A. N. Cleland and M. L. Roukes, Appl. Phys. Lett. 69, 2653 (1996).

[8] V. L. Gurevich, Sov. Phys. JETP 37, 51 (1960); 37, 1190 (1960).

[9] V. M. Kontorovich, Sov. Phys. JETP 32, 1146 (1971); Sov. Phys. Usp. 27, 134 (1984).

[10] Sh. M. Kogan and A. Ya. Shulman, Sov. Phys. JETP 29, 467 (1969).

[11] V. B. Fiks, Sov. Phys. JETP 48, 68 (1978).

[12] The average force is nonzero near a surface, where $\bar{n}$ is spatially dependent. See, for example, M. I. Kaganov and V. B. Fiks, Sov. Phys. JETP 46, 393 (1977).

[13] S. Hess and W. Köhler, Formeln zur Tensor-Rechnung (Palm \& Enke, Erlangen, 1980).

[14] K. E. Nagaev, Phys. Lett. A 169, 103 (1992).

[15] K. E. Nagaev, Phys. Rev. B 57, 4628 (1998).

[16] While the force noise $\mathcal{P}$ is white at frequencies below the inverse scattering time, the displacement noise $\mathcal{P}_{u}(\omega)$ has the resonant frequency profile $\mathcal{P}_{u}=\mathcal{P}(Q / M)^{2}\left[\omega^{2} \omega_{0}^{2}+\right.$ $\left.Q^{2}\left(\omega^{2}-\omega_{0}^{2}\right)^{2}\right]^{-1}$ (with $\omega_{0}, Q$, and $M$ the resonance frequency, quality factor, and active mass, respectively, of the oscillator). A measurement of the mean-squared displacement $\overline{u^{2}}$ (as in Ref. [3]) amounts to a measurement of the integrated spectral density, hence $\overline{u^{2}}=\mathcal{P} Q / 4 M^{2} \omega_{0}^{3}$.

[17] L. D. Landau and E. M. Lifshitz, Theory of Elasticity (Pergamon, Oxford, 1959).

[18] For a more precise theory of charge fluctuations, see M. H. Pedersen, S. A. van Langen, and M. Büttiker, Phys. Rev. B 57, 1838 (1998).

[19] Ya. M. Blanter and M. Büttiker, Phys. Rep. 336, 1 (2000). 